\title{
Development and Validation of Multiparametric Mri- based Nomogram for Predicting Occult Metastasis Risk in Early Tongue Squamous Cell Carcinoma
}

\section{Pensiri Saenthaveesuk}

Sun Yat-Sen University Guanghua School of Stomatology

Le Yang

Sun Yat-Sen University Guanghua School of Stomatology

Bin Zeng

Sun Yat-Sen University Guanghua School of Stomatology

Meng Xu

Sun Yat-Sen University Guanghua School of Stomatology

\section{Simon Young}

University of Texas Health Science Center at Houston

\section{Guiqing Liao}

Sun Yat-Sen University Guanghua School of Stomatology

\section{Yujie Liang ( $\square$ yujie0350@126.com )}

Sun Yat-Sen University Guanghua School of Stomatology

\section{Research}

Keywords: Magnetic resonance imaging, Neoplasm invasion, Tumor Thickness, Tongue cancer, Squamous cell carcinoma, Neoplasm metastasis, Nomograms, Cohort studies

Posted Date: June 17th, 2020

DOI: https://doi.org/10.21203/rs.3.rs-35585/v1

License: (c) (i) This work is licensed under a Creative Commons Attribution 4.0 International License. Read Full License 


\section{Abstract}

Background: Nomograms are currently used in predicting individualized outcomes in clinical oncology of several cancers. However, nomograms for evaluating occult nodal metastasis of patients with squamous cell carcinoma of lateral tongue (SCCLT) have not been widely investigated for their functionality. This retrospective cohort study was designed to address this question.

Methods: This study was divided into primary and validation cohorts. The primary cohort comprised 120 patients diagnosed between 2012 and 2017, whereas the validation cohort included 41 patients diagnosed thereafter. The diagnostic value of multiparametric MRI, including radiologic tumor thickness threshold (rTTT) in three-dimensions, paralingual distance, and sublingual distance were investigated. A nomogram was developed based on stepwise logistic regression of potential predictors associated with nodal metastasis in the primary cohort and then tested for predictive accuracy in the validation cohort using area under the curve (AUC) and goodness-of-fit tests.

Results: Multivariate analysis, tumor size (odd ratio [OR] 15.175, 95\% confidence interval [CI] 1.436160.329, $P=0.024$ ), rTTT (OR 11.528, 95\% Cl 2.483-53.530, $P=0.002$ ), paralingual distance (OR 11.976, $95 \% \mathrm{Cl} 1.981-72.413, P=0.005$ ), and tumor location (OR 6.311, 95\% Cl 1.514-26.304, $P=0.011$ ) were included in the nomogram to predict the likelihood of having cervical metastasis. A nomogram cutoff value of 210 points (sensitivity $93.8 \%$, specificity $87.5 \%$ ) was significantly different to classify the patients metastasis risk group $(P<0.001)$. Nomogram showed predictive accuracy with AUC 0.881 (95\% $\mathrm{Cl} 0.779-0.983, P<0.001)$ and good calibration after the validation.

Conclusions: A preoperative nomogram incorporating multiparametric MRI demonstrated good prediction and performed adequately in our study. Three-dimensional assessment of occult metastasis risk value obtained from this nomogram can assist in preoperative decision making whether to manage with neck surgery for individual patients with early-stage SCCLT. The probability of nodal metastasis tended to be greater than $20 \%$ in patients with high metastasis risk or nomogram total score $>210$ points.

\section{Background}

Management of patients with clinically node-negative (cNO) early-stage oral cancer remains controversial[1-6]. Particularly, early oral tongue cancer has a strong tendency to metastasize to regional lymph nodes subclinically, with metastasis rates between $23 \%$ and $40 \%[7-10]$. Among various factors influencing prognosis in oral cancer patients, pathologic depth of invasion (pDOI) is currently the most accurate predictor of occult metastasis and should be used to guide decision-making according to the newly updated American Joint Committee on Cancer (AJCC), 8th Edition. However, pDOI which is useful for deciding postoperative adjuvant treatment and conducting follow-up, cannot be accurately assessed in the pretreatment evaluation. 
There are severalimaging methods available for preoperative evaluation.More recent studies suggest that the high resolution of magnetic resonance imaging (MRI) is reliable optimally depicts soft tissue tumor extension, and is useful to assess tumor thickness in predicting cervical lymph node metastasis[11-13]. Previous studies have reported that MRI-assessed tumor thickness is correlated strongly and directly with pathologic thickness[14-22]. However, various MRI techniques have been proposed to assess the tumor thickness of tongue tumors. There are some controversies regarding the significance of a precise clinically optimal cutoff value of the MRI-assessed tumor thickness. Considering aborderline MRIassessed tumor thickness cutoff value for cervical lymph node involvement has a broader range varying from $4 \mathrm{~mm}$ to $14.5 \mathrm{~mm}$, the optimal cutoff value has not been established and there are no proven techniques toproperly measureMRl-assessed tumor thickness.

Nomograms are currently the most reliable discriminating tools for predicting individualized outcomes in clinical oncology to develop a treatment plan and follow-up strategies in several cancers[23-25]. Recently, previous studies have reported nomograms predicting long-term survival rate and predict cervical metastasis in head and neck cancer patients[26-30]. To date, a nomogram for predicting the likelihood of cervical lymph node metastasis pre-operatively of individual patients with early-stage of squamous cell carcinoma of lateral tongue (SCCLT) has not been widely investigated and no study has verified functionality of a MRI-based nomogram.

Additionally, solid cancer cells typically spread in different planes to invade surrounding structures as three-dimensional features. Studies interested in this aspect have reported valuable results. Kwon et al. [21]conducted the three-dimensional (3D) measurement of MRI-assessed tumor thickness. They reported MRI-assessed tumor thickness in 3D was a potential predictive indicator to evaluate occult cervical metastasis. Likewise, Okura et al.[16] reported that tumor thickness and the distance to the paralingual space assessed in the MRI coronal view had a predictiveability for nodal metastasis in SCCLT.

Presumably, cancer cell metastasis occurred through lymphovasculardrainage under the intrinsic tongue muscles to the cervical lymph node.

Therefore, the presentstudy designed two cohort series and aimed to (1) evaluate the ability and identify an optimal cutoff value of multiparametric MRI in three-dimensions to predict the presence of cervical lymph node involvement, (2) establishan accurate nomogram based on MRI forpreoperative assessment of individual patient occult metastasisriskand (3) determine the validity of a MRI-based nomogram.

\section{Methods}

\section{Patient selection}

\section{Primary and validation cohorts}

This study was approved by the Institutional Review Board of Hospital of Stomatology, Sun Yat-sen University. We performed a retrospective review of 120 patients who received a diagnosis of cN0 early- 
stage SCCLT between January 2012 and November 2017 and had not received treatment for the same diagnosis before hospitalization; these patients were included in the primary cohort. To examine the generalizability of the nomogram, external validation was performed using a separate cohort of 41 consecutive patients presenting in the same institution between December 2017 and August 2018 who had complete data to score all the variables in the nomogram. No data from the validation cohort were used to derive the nomogram, and no data from the primary cohort were used to validate the nomogram. All flat tongue tumors were included in this study. Only patients who had been examined through preoperative MRI at the medical diagnosis center, Sixth Affiliated Hospital of Sun Yat-sen University, were included in our study.

All patients received surgery as definitive treatment, which included patients who received primary resection with elective neck dissection(END) and adjuvant therapy as required. Adjuvant radiotherapy was considered for patients with positive lymph nodes, perineural invasion (PNI), and lymphovascular invasion ( $\mathrm{LVI}$ ). Concurrent chemoRT was indicated in patients with extranodal extension or positive surgical margins. All patients who were followed up for at least 12 months were included in our study. Patients were evaluated every 1-3 months in the first year, every 2-6 months in the second year, every 48 months in years $3-5$, and every 12 months thereafter.

\section{MRI and pathologic parameters measurement}

MRI was performed using the 1.5-T system Siemens MRwith the following parameters: field of view: $215.3 \mathrm{~mm}$, acquisition matrix: $156 \times 256$, and slice thickness: $3 \mathrm{~mm}$. Preoperative multiparametric MRI were measured in three-dimensions (axial, coronal, and sagittal views) with spin-echo sequences on contrast-enhanced, fat-suppressed T1WI (TR/TE:574 ms/9.9 ms) were obtained. A single-blinded reviewer (a specialist in the field of head and neck radiology) provided independent interpretation without knowledge of the clinicopathologic findings. MRI-assessed tumor thickness was initially determined byidentifying the plane of tumor thickness, with a reference line drawn as the longest tumor diameter. The length was measured from the point of maximal tumor projection perpendicular to the reference line to the deepest infiltration point, represented as MRl-assessed tumor thickness. The same measurement method was used to determineMRI-assessed tumor thickness in each plane. Consequently, axial tumor thickness (aTT), coronal tumor thickness (cTT) and sagittal tumor thickness (sTT) were defined as MRIassessed tumor thickness in the axial, coronal, and sagittal view, respectively as described in Figure 1.In this study, to improve the accuracy of MRI-assessed tumor thickness in detecting of cervical metastasis, we defined if a patient met a "radiologic tumor thickness threshold (rTTT)" with a predictor representing MRI-assessed tumor thickness in 3D. This evaluated the values obtained from aTT, cTT and STT measurements for each patient and could be categorized into two groups: "minimal" and " significant" rTTT, depending on how many MRI-assessed tumor thicknesses exceed their cutoff values. Minimal rTTT was defined as only one orientation of MRI thickness (either aTT, cTT or STT) found to be greater than its defined cutoff value or no MRI thicknesses exceeding their cutoff values. Significant rTTT was defined as at least two orientations of MRI thickness (either aTT, cTT or sTT) exceeding their cutoff values. 
Paralingual distance (PLD) and sublingual distance (SLD)were determinedas described by Okura et al.[16]in coronal T1-weighted MRI and are illustrated in Figure 1B. The inferior lateral aspect of the tongue with high signal intensity represented the sublingual space. The SLD was determined by measuring the vertical distance between the inferior border of the tumor and the sublingual space. The thin high signal intensity are extending from the medial aspect of sublingual space along the genioglossus or hyoglossus muscle (low signal intensity area) tothe deep lingual artery was determined to be theparalingual space. PLD was defined as the distance between the medial border of the tumorand the paralingual space.For lesions in which tumor extension occurred beyond the deep lingual artery and the sublingual space, PLD and SLD were determined to be $0 \mathrm{~mm}$.

Surgical specimens of the tongue were fixed in $20 \%$ formalin, embedded in paraffin, and then stained with hematoxylin and eosin for examination through light microscopy. This study included only flat tumors in which the mucosal plane was at the same level as the tumor. Then the pathologic depth of invasion and tumor thickness could be measured identically $(\mathrm{pDOI}=\mathrm{pTT})[13]$. One experienced pathologist who was unaware of the MRI findings oriented the specimen and routinely performedmeasurement on tumor depth of invasion.Only pathologic thicknessin the axial view was available in our institution during the study period. Postoperative pathological parameters of celldifferentiation, presence of lymphovascular invasion (LVI), muscular invasion and perineural invasion (PNI) were also investigated.

\section{Statistical analysis}

All statistical analyses were conducted using SPSS (version 21; Chicago, IL, USA) and R software (version 3.6). Descriptive variables of patient characteristics and multivariableMRI were analyzed. Receiver operating characteristic (ROC) curve analysis was conducted to identify the optimal cutoff value of each MRI parameterin evaluating cervical metastases. ROC curve was drawn on the basis of the sensitivity and specificity, and the area under the curve (AUC) was also calculated. Pearson correlation coefficient was used to assess the correlation between MRI and pathological thickness. Diagnostic accuracy, including sensitivity, specificity, positive predictive value (PPV), negative predictive value (NPV), positive likelihood ratio (+LR), and negative likelihood ratio (-LR), in correctly identifying positive and negative neck nodes was evaluated.

The study population was divided into primary and validation cohorts. A binary logistic regression predictive model was used to construct the nomogram in the primary cohort, which was then used to verifythe predictive accuracy in the validation cohort. On multivariate logistic regression, we included multiple variables that had significant associations with occult nodal metastasis in the final nomogram. Nomogram performance was quantified in terms of discrimination and calibration in both cohorts. ROC curve methodology was used to assess the discrimination power and total score cutoff value of the nomogram. The AUC was used to quantify the predictive accuracy of nomogram.Calibration was assessed graphically by plotting the relationship between actual and predicted probabilities by using the Hosmer goodness-of-fit test. Model validation was performed using the bootstrapping method (1000 
replications) to quantify our modeling strategy. A value of $P<0.05$ was considered to indicate statistical significance.

\section{Results}

\section{Patient characteristics}

The characteristics of patients in both cohorts are summarised in Table 1. Of the 120 patients in the primary cohort, $49.2 \%(59 / 120)$ had cNO T1 tumors, and 50.8\%(61/120) had T2 tumors. The treatment modality was recorded for all patients, 120 patients were treated with primary resection and END, 26.7\% $(32 / 120)$ of whom had nodal metastasis $(\mathrm{pN}+)$. The mean values of aTT, cTT, sTT, PLD and SLD was $9.89 \pm 4.07 \mathrm{~mm}, 13.44 \pm 5.63 \mathrm{~mm}, 14.35 \pm 6.52 \mathrm{~mm}, 4.38 \pm 2.47 \mathrm{~mm}$ and $3.80 \pm 2.94 \mathrm{~mm}$, respectively. The mean follow-up time was 45 months (18-82 months). Recurrence or metastasis occurred in $15 \%$ $(18 / 120)$ : at the primary site in $5 \%(6 / 120)$, in the neck in $4.17 \%(5 / 120)$ and $5.83 \%(7 / 120)$ had distant metastasis in the primary cohort.

For postoperative pathologic results, the mean value of pathologic thickness was $8.06 \pm 3.47$ $\mathrm{mm}$.Pearson's correlation coefficient showed a strong positive correlation between pathologic thickness and MRI thickness in mediolateral direction (aTT) with $0.836(P<0.001)$. Furthermore, we found a significant difference in predicting cervical lymph node status when incorporating $\mathrm{pDO}$ according to the AJCC ( $8^{\text {th }}$ edition), no occult metastasis was detected in $\mathrm{pDOI} \leq 5 \mathrm{~mm}$, metastasis rate was $25.3 \%$ in pDOI $>5 \mathrm{~mm}$ to $\leq 10 \mathrm{~mm}$ and metastasis rate was $78.6 \%$ in pDOl> $10 \mathrm{~mm}(P<0.001)$. The presence of PNI was also significantly different according to nodal status $(P=0.046)$.In contrast, cancer cell differentiation $(P=0.824)$, presence of $\operatorname{LVI}(P=0.663)$ and muscular invasion $(P=0.304)$ were not significantly different for predicting nodal metastasis.

\section{Identifying cutoff values of MRI parameters to estimatepN+}

In this study, we focused on assessment of occult metastasis risk in early-stageSCCLT by evaluation of MRI parameters in three-dimensional planes. ROC curve analysis was applied to calculate the optimal cutoff value of the preoperative MRI parameters for identifying regional lymph node metastasis. The ability and effectiveness of each parameter were analyzed using their cutoff value to calculate sensitivity, specificity, PPV, NPV and likelihood ratio. The effect of cutoff value impacting the diagnostic accuracy is presented in Table 2 and the ROC curve regarding the usedcutoff value of predictors to assess subclinical nodal metastases are also shown in Figure2.

ROC curve analysis indicated that the aTTof $8.5 \mathrm{~mm}$, cTT of $11 \mathrm{~mm}$ and sTT of $12 \mathrm{~mm}$ might be the optimal threshold value for predicting cervical lymph node metastases, with the AUC being $0.895,0.867$ and 0.916 , respectively. Notably, the predictivevalue of aTT with $8.5 \mathrm{~mm}$ cutoff value was $84.4 \%$ sensitivity, 86.4\% specificity, 69.2\% PPV, 93.8\% NPV, +LR 6.00 and -LR $0.19(P<0.001)$. Likewise the diagnostic value of sTT with $14 \mathrm{~mm}$ cutoff value was $90.6 \%$ sensitivity, $81.8 \%$ specificity, $64.4 \%$ PPV, $96 \%$ NPV, +LR 5.06 and -LR 0.11( $P<0.001)$. Additionally,the incidence of nodal metastasis was higher in 
patients with significantrTTT than in patients with minimalrTTT ( $65.1 \%$ versus $5.2 \%, P<0.001)$. The ROC curves also reflected that PLD of $5 \mathrm{~mm}$ and SLD of $4 \mathrm{~mm}$ might be the optimal decision threshold related to nodal metastases, with the AUC being 0.759 and 0.616 , respectively.

\section{Development of multiparametric MRl-derived nomogram for predicting of individualized occult metastasis}

A univariate analysis was initially performed to evaluate the association between each variable and lymph node metastases. Besides,rTTT, PLD and SLD based on MRI, some other risk variables were evaluated, including sex, age, tumor size (T1 or T2), pathologic differentiation (well or moderate-poor), and tumor location (anterior-middle or posterior). The probability of having lymph node metastasis in each variable was estimated. Then, all variables with a $P$ value $<0.05$ in the univariate analysis were entered in a multivariate logistic regression analysis model by using a backward condition method and remaining variables were used to create a nomogram to predict the probability of cervical lymph nodepositive disease in the primary cohort. Multivariate logistic regression analysis demonstratedthat tumor size (OR 15.175, 95\% Cl 1.436-160.329, $P=0.024$ ), rTTT (OR 11.528, 95\% Cl 2.483-53.530, $P=0.002$ ), PLD (OR 11.976, 95\% Cl 1.981-72.413, $P=0.005$ ), and tumor location (OR 6.311, 95\% Cl 1.514-26.304, $P=$ 0.011 ) emerged as significant predictors to indicate the existence oflymph node metastasis of early-stage SCCLT(Table 3).

Ultimately, four potential predictors were presented in the final nomogram that identify the occult metastasis risk and predict the patient on the likelihood of cervical nodal involvement (Figure 3). Nomogram was characterized by one scale corresponding with each variable, a score scale, a total score scale, and a probability scale. The top row shows the point assignment for each predictor. The metastasis risk predictors in order of their listing in the nomogram are: tumor size, tumor location, rTTT and PLD which is each predictorrepresent a point value based on the tumor characteristics (row 2-4). The points assigned to each of the four predictors are summed, and the total points indicated in row 5 . The bottom row shows the probability of the patient having cervical lymph node metastasis.

\section{Nomogram use for classifying patient metastasis risk group}

ROC curve analysis was also applied to identify the optimal cutoff value of total score obtained from the nomogram in the primary cohort.The ROC curve analysis indicated that the optimal cutoff value of nomogram total score of 210 points was significantly different to discriminate the patients likelihood of having cervical lymph node metastasis preoperatively. With regard to this cutoff value, patient may be categorized as having a low occult metastasis risk depending upon their total risk score less than 210 points. While, total risk scores more than 210 points, patients were stratified in a high occult metastasis risk. The possibility of metastasis ratehigher $20 \%$ whentotal score more than cutoff value of 210 points was presented.

In the primary cohort, the MRI-based nomogram demonstrateda predictive accuracy of predicting individualized positive cervical lymph nodes, resulting in excellent discrimination power of the AUC 
0.952with $95 \% \mathrm{Cl} 0.917-0.987, P<0.001$ in Figure 4A. The sensitivity, specificity, PPV, and NPV of the nomogram using the cutoff value of 210 points were $93.8 \%, 87.5 \%, 73.2 \%$ and $97.5 \%$, respectively $(P<$ 0.001) The Hosmer-Lemeshow goodness-of-fit test indicated that the nomogram was well-calibrated and the slope hadno apparent departure from perfect fit $(P<0.001)$. The calibration plot showed good agreement between the prediction and metastasis risk observation (Figure 4C).

\section{Clinical validation of MRI-based nomogram}

Validation cohort analysis was carried out on41 patients who were diagnosed as having T1 tumors $51.20 \%(21 / 41)$, and T2 tumors $48.80 \%$ (20/41). All patients were treated with primary resection and END, $29.3 \%(12 / 41)$ of whom had cervical node metastasis $(\mathrm{pN}+)$.Considering the nomogram cutoff value of 210 points generated the patients into metastasis risk groups, 25 patients $(60.98 \%)$ with total score obtained from nomogram less than 210 points had a low metastasis risk. On the other hand, 16 patients (39.02\%) with total score obtained from nomogram more than 210 points had a highmetastasis risk.Incidence of nodal metastasisdemonstrated a statistically significant difference between the low and high metastasis risk groups $(P=0.001)$. The incidence of nodal metastasis was higher in the high metastasis risk group (62.5\%), than in the low metastasis risk group (8.0\%)

Validation of the nomogram was conducted both internally and externally, as presented in Figure 4. In the entire validation cohort, theROC curve analysis reflected the MRI-based nomogram demonstrated excellent prediction performance with high predictive value of AUC $0.881,95 \% \mathrm{Cl} 0.779-0.983(P<0.001)$ in Figure 4B. Additionally, we conducted a diagnostic test by using nomogram cutoff value of 210 points, the result showed sensitivity of $83.3 \%$, specificity of $79.3 \%$, PPV of $62.5 \%$, and NPV of $92.0 \%$ in the validation cohort. The calibration curves of the nomogram in the validation cohorts also wereplotted, and the slope was close to 1 , indicating that the nomograms were well calibrated in Figure 4D.

\section{Discussion}

Cancer patients will present with several clinical characteristics that can have either positive or negative influences on predicting the likelihood of the occult metastasis risk. Reviewing these clinical aspects of patient will help to generate an initial metastasis risk profile. Nomograms are widely used in pretreatment evaluation of several cancers because the nomogram is useful scoring and reproducible method. This study demonstrated that the multiparametric MRI-based nomogram is good discrimination tool which can be used to predict individualized metastasis outcome for preoperative evaluation, and high NPV results of the nomogram may help to prevent unnecessary neck surgery and morbidity in patients with early-stage SCCLT. Study of Swawantet al.[29]also proposed a nomogram for predicting the risk of neck node metastasis in pathologically node-negative in 103 of T1-T4 oral cavity carcinoma patients by using a combination of protein expression, ultrastructural alterations and clinicopathological parameters. Likewise, Jiang et al.[30] developed the nomogram to predict the probability of occult cervical lymph node metastasis before surgery in patients with cNO SCC of the tongue with high (C-index $=0.846)$ and good calibration. 
Specific features of our MRI-based nomogram includeuse of four potential predictors of rTTT, PLD, tumor size and tumor location which is a set of continuous scale representation of three-dimensional evaluation. This comprehensive evaluationwould increase the predictive power of the nomogram. Moreover, we could achieve the nomogram optimal cutoff value of 210 points with high sensitivity of $93.8 \%$ and specificity of $87.5 \%$ for classifying occult nodal metastasis risk in our study populations. Then we tested the predictive accuracy of nomogram to identify patients at high risk for pre-operative, clinically undetectable cervical lymph node metastasis in the validation cohort. The reliable results showed that the nomogram had a good predictive ability with an AUC of 0.881 , sensitivity of $83.3 \%$, specificity of $79.3 \%$ and well-generalized calibration, which demonstrated a suitable degree of accuracy and quantified ability of the nomogram to discriminate between patients with and without cervical lymph node involvement.

A predicted probability of metastasis rate $>20 \%$ was presented in patients with high metastasis risk group who obtained total score from the nomogram more than 210 points. Clinical T2 and tumor located in posterior of tonguewere commonly found in high metastasis risk group. In study of Feng et al. [4] suggests that END should be a preferred treatment strategy for tongue carcinoma in stage T2. Similar to resultsfrom a study of Sagheb et al.[31]and Jiang et al.[30] reported that the probability of cervical metastases rate of tongue SCC located in the posterior third region was significantly higher than when tumor was located in the anterior two-third of the tongue. Probably the posterior area of the tongue is supplied with plenty of lymphatic drainage to the superficial and deep cervical lymph nodes and had a greater chance of developing positive neck node disease.

As a matter of fact cancer cells typically spread in different planes to invade surrounding structures, and evaluation in three-dimensions is a more accurate prediction, reflecting the entire tumor extension.

Therefore, we selected the technique measurement similar to the study of Kwon[21]to investigate MRIassessed tumor thickness and identified their cutoff value in three directions. A logistic regression model showed that significantrTTTresulted in a more accurate metric to reflect the extent of cancer invasion and comprehensive interpretation of tumor characteristics than minimalrTTT. We found that MRI-assessed tumor thickness extending beyond the cutoff value in at least two directions highly increased the nodal metastasis rate. Based on the results showed rTTT represent the better result of MRI-derived thickness and practically adopted in determining metastasis outcome.

Reviewing currently reliable evidence the most common MRI-assessed tumor thickness cutoff valuesavailable range from $7.5 \mathrm{~mm}$ to $12.3 \mathrm{~mm}[11,12,16-18,20,21]$. Hence, It is difficult to compare between studies because previous studies have measured MRI-assessed tumor thicknesses from various techniques and different directions. However, we found the clinically useful cutoff value of MRI-assessed tumor thickness was $8.5 \mathrm{~mm}, 11 \mathrm{~mm}$ and $12 \mathrm{~mm}$ in axial, coronal and sagittal planes, respectively.Particularly, the predictive power of aTT was $84.4 \%$ sensitivity, $86.4 \%$ specificity and sTT was $90.6 \%$ sensitivity, $81.8 \%$ specificityto detect cervical lymph node involvement $(P<0.001)$. Our result reported cutoff value consistent with previous studies of MRI thickness in tongue and oral cancer[14-22]. As study by Hu et al.[20]reported MRI-assessed tumor thicknessof $8.5 \mathrm{~mm}$ wasusefulinpredictingtheprognosisoflymph node metastases for 46 patients with tonguesquamous cell 
carcinomas.Park et al.[18], who studied 49 patients with tongue carcinoma and reported the relationship between MRI invasion depth and nodal metastasis with a cutoff value of $9.5 \mathrm{~mm}$ on T1WI. Jung et al.[17], who reported on 50 patients with T1-T2 tongue carcinoma and recommended MRI invasion depth cutoff values of $11 \mathrm{~mm}$ and $12 \mathrm{~mm}$ for T1-and T2-weighted cases, respectively, showed significant correlation with nodal metastasis. This was similar in result to Kwon et al.[21] They reported MRI-assessed tumor thicknesscutoff value of $12.3 \mathrm{~mm}$ in sagittal plane significant different to evaluate cervical metastasis.

Recently, pDOI has become an essential predictor of occult nodal metastasis[31-35]. Several studies have emphasized the correlation between pathological thickness and MRI thickness[14-22]. The current study confirmed a strong correlation between MRI and pathological thickness [ $r=0.836]$. The eighth edition of the AJCC proposed incorporating pDOI into three groups ( $5 \mathrm{~mm}$, from $>5$ to $10 \mathrm{~mm}$, and $>10 \mathrm{~mm}$ )[32]. We found that none of our patients whose pDOI was less than $5 \mathrm{~mm}$ detected nodal metastasis, while all whose $\mathrm{pDOI}$ exceeded $5 \mathrm{~mm}$ the occult metastasis incidence rate was occurred $>20 \%$. Our result corresponding with previous studies, meta-analysis of Huang et al.[34]reported a statistically significant difference between the $4 \mathrm{~mm}$ and $5 \mathrm{~mm}$ thickness cutoff values for cervical metastasis in oral carcinoma. Previous studies in tongue carcinoma also stated that higher incidence of nodal metastasis was detected when $\mathrm{pDO}>5 \mathrm{~mm}[2,17,33]$. This is likely because intrinsic muscle of tongue is a poor barrier to tumor spread drainage to cervical lymphatics system.

In addition, other MRI predictors describing tumor extension included SLD and PLD. With regard to our result, SLD does not seem useful for prediction the existence of lymph node metastasis. Multivariate analysis showed PLD with a cutoff value of $5 \mathrm{~mm}$ was a more reliable predictor related to nodal metastasis than SLD and was included in the nomogram. Another valuable finding is that the risk of nodal metastasis was $>20 \%$ in patients with PLD $\leq 5 \mathrm{~mm}$ because increasing of tumor extension in close proximity to the lingual lymph nodes and lingual vessels on the lateral aspect of tongue would have greater chances to develop metastasis. Lingual lymph node metastasis have been reported in oral squamous cell carcinoma[36].Similar result to study of Okura et al.[16]reported that in 43 patients with SCCT, PLD was a significant predictor of lymph nodal metastases. END decision might be based on PLD $<5.2 \mathrm{~mm}$. As previous mentioned, PLD was an outstanding predictor that could also represent tumor extension and might have a role in predicting nodal metastasis.

Our study has some limitations. First, the nomogramswere constructed using retrospective data, which might introduce the risk of treatment selection bias. Also, sample size in our study was limited to the oral tongue sub-site and single center analysis. Therefore, these nomograms must be further validated in a larger prospective cohort in other sub-sites, and accurate risk estimates are required for the clinical trial design. Second, the follow-up period in this study should have been longer to provide more accurate in predictions. Third, standardized technique and better resolution of imaging to measure MRI thickness should be clarified and used to identify the precise cutoff value necessitating further study to confirm our findings.

\section{Conclusions}


In summary, the nomogram demonstrated predictive accuracy of AUC 0.952 in the primary and AUC 0.881 in the validation cohorts. The effect of three-dimensional evaluation of MRI predictors impacted the predictive accuracy of preoperative nomogram. Assessment of occult metastasis risk value obtained from MRI-based nomogram can assist in preoperative decision making whether to manage with neck surgery for individual patients with early-stage SCCLT. The probability of nodal metastasis tended to be greater than $20 \%$ in patients with high metastasis risk or nomogram total score $>210$ points, and these patients should be treated with prophylactic neck dissection. We concluded that the multiparametric MRIbased nomogram with reasonable accuracy and discrimination was generated and externally validated to predict individualized metastasis outcome.

\section{List Of Abbreviations}

SCCLT squamous cell carcinoma of lateral tongue

cNO clinically node-negative

AJCC American Joint Committee on Cancer

MRI magnetic resonance imaging

3D three dimensions

rTTT radiologic tumor thickness threshold

aTT axial tumor thickness

CTT coronal tumor thickness

STT sagittal tumor thickness

PLD paralingual distance

SLD sublingual distance

pDOI pathologic depth of invasion

PTT pathologic tumor thickness

$\mathrm{PNI} \quad$ perineural invasion

LVI lymphovascular invasion

ROC receiver operating characteristic

AUC area under the curve 


$\begin{array}{ll}\text { PPV } & \text { positive predictive value } \\ \text { NPV } & \text { negative predictive value } \\ \text { LR+ } & \text { positive likelihood ratio } \\ \text { LR- } & \text { negative likelihood ratio } \\ \text { OR } & \text { odds ratio } \\ \text { Cl } & \text { confidence interval } \\ \text { pN+ } & \text { positive neck-node } \\ \text { END } & \text { elective neck dissection }\end{array}$

\section{Declarations}

Ethics approval and consent to participate:The present study was approved by the Ethic Committee of Institution Research of Hospital of Stomatology, Sun Yat-sen University(No.ERC-2016-28). Additionally, the written informed consent was obtained from the patients.

Consent for publication:Not applicable

Availability of data and materials: The datasets used and/or analysed during the current study are available from the corresponding author on reasonable request.

Competing interests:The authors declare that they have no competing interests

Funding: This work was supported by the 5010 Projects of Clinical Study, Sun Yat-sen University (No.2010018); Guangdong Financial Fund for High-Caliber Hospital Construction; and the National Natural Science Foundation of China (No.81602384).

\section{Authors' contributions:}

Pensiri Saenthaveesuk: Conceptualization, data curation, formal analysis, investigation, methodology, project administration, resources, software, supervision, validation, visualization, writing - original draft, and writing - review and editing.

Le Yang: Conceptualization, Data curation, methodology, project administration, validation, writing original draft, and writing - review and editing.

Bin Zeng: Formal analysis, resources, software, and writing - review and editing.

MengXu: Data curation, formal analysis, investigation, resources, and writing - review and editing. 
Simon Young: Conceptualization, formal analysis, methodology, validation, visualization, and writing review and editing.

Guiqing Liao: Conceptualization, investigation, methodology, funding acquisition, project administration, resources, supervision, validation, visualization, and writing - review and editing.

Yujie Liang: Conceptualization, investigation, methodology, funding acquisition, project administration, resources, supervision, validation, visualization, and writing - review and editing.

All authors read and approved the final manuscript.

Acknowledgements: Not applicable

\section{References}

1. Lim S, Zhang S, Ishii G, Endoh Y, Kodama K, Miyamoto S, Hyashi R, Ebihara S, Cho H, Ochiai A: Predictive markers for late cervical metastasis in stage I and II invasive squamous cell carcinoma of the oral tongue. Clin Cancer Res 2004, 10(1 Pt 1):166-172.

2. Pentenero M, Gandolfo S, Carrozzo M: Importance of tumor thickness and depth of invasion in nodal involvement and prognosis of oral squamous cell carcinoma: a review of the literature. Head Neck 2005, 27(12):1080-1091.

3. Mücke T, Mitchell D, Wagenpfeil S, RitschI L, Wolff K, Kanatas A: Incidence and Outcome for Patients With Occult Lymph Node Involvement in T1 and T2 Oral Squamous Cell Carcinoma: A Prospective Study BMC Cancer 2014, 14:346.

4. Feng Z, Li J, Li C, Guo C: Elective Neck Dissection Versus Observation in the Management of Early Tongue Carcinoma With Clinically Node-Negative Neck: A Retrospective Study of 229 Cases $\mathrm{J}$ Craniomaxillofac Surg 2013, 42(6):806-810.

5. Mizrachi A, Migliacci J, Montero P, McBride S, Shah J, Patel S, Ganly I: Neck recurrence in clinically node-negative oral cancer: 27-year experience at a single institution. Oral Oncol2018, 78:94-101.

6. Cao Y, Wang T, Yu C, Guo X, Li C, Li L: Elective Neck Dissection Versus Wait-and-Watch Policy for Oral Cavity Squamous Cell Carcinoma in Early Stage: A Systematic Review and Meta-Analysis Based on Survival Data J Oral Maxillofac Surg 2019, 77(10):2154-2167.

7. Ross G, Soutar D, MacDonald D, Shoaib T, Camilleri I, Robertson A: Improved Staging of Cervical Metastases in Clinically Node-Negative Patients With Head and Neck Squamous Cell Carcinoma. Ann Surg Oncol 2004, 11(2):213-218.

8. Keski-Santti H, Atula T, Tornwall J, Koivunen P, Makitie A: Elective neck treatment versus observation in patients with T1/T2 N0 squamous cell carcinoma of oral tongue. Oral Oncol 2006, 42(1):96-101.

9. Mair M, Shetty R, Nair D, Mathur Y, Deshmukh A, Thiagarajan S, Pantvaidya G, Lashkar S, Prabhash $\mathrm{K}$, Chaukar $\mathrm{D}$ et al: Depth of invasion, size and number of metastatic nodes predicts extracapsular spread in early oral cancers with occult metastases. Oral Oncol 2019, 81:95-99. 
10. O'Brien C, Lauer C, Fredricks S, Clifford A, McNeil E, Bagia J, Koulmandas C: Tumor thickness influences prognosis of T1 and T2 oral cavity cancer-but what thickness? Head Neck 2003, 25(11):937-945.

11. Murakami R, Shiraishi S, Yoshida R, Sakata J, Yamana K, Hirosue A, Uchiyama Y, Nakayama H, Yamashita Y: Reliability of MRI-Derived Depth of Invasion of Oral Tongue Cancer. Acad Radio/ 2019, 26(7):e180-e186.

12. Mao M, Wang S, Feng Z, Li J, Li H, Qin L, Han Z: Accuracy of Magnetic Resonance Imaging in Evaluating the Depth of Invasion of Tongue Cancer. A Prospective Cohort Study Oral Oncol 2019, 91:79-84.

13. Weimar E, Huang S, Lu L, O'Sullivan B, Perez-Ordonez B, Weinreb I, Hope A, Tong L, Goldstein D, Irish $\mathrm{J}$ et al: Radiologic-Pathologic Correlation of Tumor Thickness and Its Prognostic Importance in Squamous Cell Carcinoma of the Oral Cavity: Implications for the Eighth Edition Tumor, Node, Metastasis Classification. AJNR Am Neuroradio/2018, 39(10):1896-1902.

14. Iwai H, Kyomoto R, HaOKawa S, Lee S, Yamashita T: Magnetic resonance determination of tumor thickness as predictive factor of cervical metastasis in oral tongue carcinoma. Laryngoscope 2002, 112(3):457-461.

15. Preda L, Chiesa F, Calabrese L, Latronico A, Bruschini R, Leon M, Renne G, Bellomi M: Relationship between histologic thickness of tongue carcinoma and thickness estimated from preoperative MRI. Eur Radiol 2006, 16(10):2242-2248.

16. Okura M, lida S, Aikawa T, Adachi T, Yoshimura N, Yamada T, Kogo M: Tumor thickness and paralingual distance of coronal MR imaging predicts cervical node metastases in oral tongue carcinoma. AJNR Am Neuroradio/ 2008, 29(1):45-50.

17. Jung J, Cho N, Kim J, Choi E, Lee S, Byeson H, Park Y, Yang W, Kim S: Significant invasion depth of early oral tongue cancer originated from the lateral border to predict regional metastases and prognosis. Int J Oral Maxillofac Surg 2009, 38(6):653-660.

18. Park J, Jung S, Joo Y, Jung C, Cho K, Kim M: Diagnostic accuracy of magnetic resonance imaging (MRI) in the assessment of tumor invasion depth in oral/oropharyngeal cancer. Oral Oncol 2011, 47(5):381-386.

19. Lwin C, Hanlon R, Lowe D, Brown J, Woolgar J, Triantafyllou A, Rogers S, Bekiroglu F, Lewis-Jones H, Wieshmann $\mathrm{H}$ et al: Accuracy of MRI in prediction of tumour thickness and nodal stage in oral squamous cell carcinoma. Oral Oncol 2012, 48(2):149-154.

20. Hu H, Cheng K, XU X, Wu F, Tyan Y, Tsai C, Shen C: Predicting the Prognosis of Oral Tongue Carcinoma Using a Simple Quantitative Measurement Based on Preoperative MR Imaging: Tumor Thickness Versus Tumor Volume AJNR Am Neuroradio/ 2015, 36(7):13382-13342.

21. Kwon M, Moon H, Nam S, Lee J, Kim J, Lee Y, Roh J, Choi S, Kim S: Clinical significance of threedimensional measurement of tumour thickness on magnetic resonance imaging in patients with oral tongue squamous cell carcinoma. Eur Radio/ 2016, 25(3):858-865. 
22. Huang C, Zhuang S, Li J, Chen S, Zhang X, Song M: Can we identify the patients with clinically T12NO oral tongue squamous cell carcinoma benefiting from neck dissection? Int J Clin Exp Med 2017, 10(2):4023-4034.

23. Liu J, Geng Q, Liu Z, Chen S, Guo J, Kong P, Chen Y, Li W, Zhou Z, Sun X et al: Development and external validation of a prognostic nomogram for gastric cancer using the national cancer registry. Oncotarget 2016, 7(24):35853-35864.

24. Ganly I, Amit M, Kou L, Palmer F, Migliacci J, Katabi N, Yu C, Kattan M, Binenbaum Y, Sharma K et al: Nomograms for predicting survival and recurrence in patients with adenoid cystic carcinoma. An international collaborative study. Eur J Cancer 2015, 51(18):2768-2776.

25. Wen J, Ye F, He X, Li S, Huang X, Xiao X, Xie X: Development and validation of a prognostic nomogram based on the log odds of positive lymph nodes (LODDS) for breast cancer. Oncotarget 2016, 7(15):21046-21053.

26. Ju J, Wang J, Ma C, Li Y, Zhao Z, Gao T, Ni Q, Sun M: Nomograms predicting long-term overall survival and cancer-specific survival in head and neck squamous cell carcinoma patients. Oncotarget 2016, 7(32):51059-51068.

27. Li Y, Zhao Z, Liu X, Ju J, Chai J, Ni Q, Ma C, Gao T, Sun M: Nomograms to estimate long-term overall survival and tongue cancer-specific survival of patients with tongue squamous cell carcinoma. Cancer Med 2017, 6(5):1002-1013.

28. Fakhry C, Zhang Q, Nguyen-Tan P, Rosenthal D, Weber R, Lamber L, Trotti Ar, Barrett W, Thorstad W, Jones $C$ et al: Development and Validation of Nomograms Predictive of Overall and Progression-Free Survival in Patients With Oropharyngeal Cancer. J Clin Oncol 2017, 35(36):4057-4065.

29. Swawant S, Dongre H, Ahire C, Sharma S, Kannan S, Mahadik S, Chaukar D, Lukmani F, Patil A, D'Cruz A et al: A Nomogram for Predicting the Risk of Neck Node Metastasis in Pathologically NodeNegative Oral Cavity Carcinoma Oral Dis 2017, 23(8):1087-1098.

30. Jiang Q, Tang A, Long S, Qi Q, Song C, XIn Y, Zhang C, Cao Z, Zhang J: Development and Validation of a Nomogram to Predict the Risk of Occult Cervical Lymph Node Metastases in cNO Squamous Cell Carcinoma of the Tongue Br J Oral Maxillofac Surg 2019, 57(10):1092-1197.

31. Sagheb K, Kumar V, Rahimi-Nedjat R, Dollhausen M, Ziebart T, Al-Nawas B, Walter C: Cervical Metastases Behavior of T1-2 Squamous Cell Carcinoma of the Tongue J Maxillofac Oral Surg 2017, 16(3):300-305.

32. Lydiatt W, Patel S, O'Sullivan B, Brandwein M, Ridge J, Migliacci J, Loomis A, Shah J: Head and Neck cancers-major changes in the American Joint Committee on cancer eighth edition cancer staging manual. CA Cancer J Clin 2017, 67(2):122-137.

33. Kane S, Gupta M, Kakade A, D'Cruz A: Depth of invasion is the most significant histological predictor of subclinical cervical lymph node metastasis in early squamous carcinomas of the oral cavity. Eur $J$ Surg Oncol 2006, 32(7):795-803.

34. Huang S, Hwang D, Lockwood G, Goldstein D, O'Sullivan B: Predictive value of tumor thickness for cervical lymph-node involvement in squamous cell carcinoma of the oral cavity: a meta-analysis of 
reported studies. Cancer 2009, 115(7):1489-1497.

35. S. T, Amit M, Zafereo M, Bell D, Weber R: Depth of Invasion as a Predictor of Nodal Disease and Survival in Patients With Oral Tongue Squamous Cell Carcinoma Head Neck 2019, 41(1):177-184.

36. Fang Q, Peng L, Qi J, Luo R, Chen D, ZHang X: Value of Lingual Lymph Node Metastasis in Patients With Squamous Cell Carcinoma of the Tongue Laryngoscope 2019, 129(11):2527-2530.

\section{Tables}


Table 1

Patient characteristics

\begin{tabular}{|c|c|c|}
\hline $\begin{array}{l}\text { Patient characteristics } \\
\mathrm{N}(\%)\end{array}$ & $\begin{array}{l}\text { Primary Cohort } \\
(\mathrm{N}=120)\end{array}$ & $\begin{array}{l}\text { Validation Cohort } \\
(\mathrm{N}=41)\end{array}$ \\
\hline \multicolumn{3}{|l|}{ Age at diagnosis [year] } \\
\hline$\cdot \leq 60$ & $87(72.5)$ & $24(58.5)$ \\
\hline$\cdot>60$ & $33(27.5)$ & $17(41.5)$ \\
\hline \multicolumn{3}{|l|}{ Gender } \\
\hline - Male & $71(59.2)$ & $23(56.1)$ \\
\hline - Female & $49(40.8)$ & $18(43.9)$ \\
\hline \multicolumn{3}{|l|}{ Tumorsize } \\
\hline$\cdot \mathrm{T} 1$ & $59(49.2)$ & $21(51.2)$ \\
\hline$\cdot \mathrm{T} 2$ & $61(50.8)$ & $20(48.8)$ \\
\hline \multicolumn{3}{|l|}{ Tumor Location } \\
\hline - Anterior/Middle & $76(63.3)$ & \\
\hline \multirow[t]{2}{*}{ - Posterior } & $44(36.7)$ & $24(58.5)$ \\
\hline & & $17(41.5)$ \\
\hline \multicolumn{3}{|l|}{ MRI parameters $[\mathrm{mm}]$} \\
\hline \multicolumn{3}{|c|}{ 1.MRI-assessed tumor thickness in 3D } \\
\hline \multicolumn{3}{|c|}{ - Tumor thickness in axial view (aTT) } \\
\hline$\leq 8.5 \mathrm{~mm}$ & $81(67.5)$ & $25(61)$ \\
\hline$>8.5 \mathrm{~mm}$ & $39(32.5)$ & $16(39)$ \\
\hline
\end{tabular}

- Tumor thickness in coronal view (cTT) $\leq 11 \mathrm{~mm}$

$>11 \mathrm{~mm}$ 69 (57.5) $11(26.8)$

$51(42.5)$ $30(73.2)$

- Tumor thickness in sagittal view (sTT)

$\leq 12 \mathrm{~mm}$

$>12 \mathrm{~mm}$ 
2. Paralingual distance (PLD)
$\leq 5 \mathrm{~mm}$
$60(50)$
24 (58.5)

$>5 \mathrm{~mm}$

$60(50)$

$17(41.5)$

3. Sublingual distance (SLD)

$$
\leq 4 \mathrm{~mm}
$$

73 (60.8)

28 (68.3)

$>4 \mathrm{~mm}$

47 (39.2)

13 (31.7)

Pathological node status

- $\mathrm{pNO}$

88 (73.3)

29 (70.7)

- $\mathrm{pN}+$

32 (26.7)

12 (29.3)

Postoperative pathological thickness*

$\cdot \leq 5 \mathrm{~mm}$

$23(19.2)$

4 (9.8)

- $>5 \mathrm{~mm}$ to $\leq 10 \mathrm{~mm}$

$83(69.2)$

$31(75.6)$

$\cdot>10 \mathrm{~mm}$

$14(11.7)$

$6(14.6)$

Cell-differentiation

- Well

$61(50.8)$

$26(63.4)$

- Moderate/Poor

$59(49.2)$

$15(36.6)$

* This study included only flat tumors in which the mucosal plane was at the same level as the tumor. Then the pathologic depth of invasion and pathological tumor thickness could be measured identically $(\mathrm{pDOI}=\mathrm{pTT})$ 
Table 2

Predictors diagnostic accuracy using cutoff value to predict presence of $\mathrm{pN}+$ cases

\begin{tabular}{|lllllll|}
\hline $\begin{array}{l}\text { Parameter } \\
\text { cutoff value }\end{array}$ & Sensitivity & Specificity & PPV & NPV & LR + & LR - \\
\hline aTT $8.5 \mathrm{~mm}$ & $84.4 \%$ & $86.4 \%$ & $69.2 \%$ & $93.8 \%$ & 6.00 & 0.19 \\
\hline cTT $11 \mathrm{~mm}$ & $87.5 \%$ & $73.9 \%$ & $54.9 \%$ & $94.2 \%$ & 3.38 & 0.16 \\
\hline STT $12 \mathrm{~mm}$ & $90.6 \%$ & $81.8 \%$ & $64.4 \%$ & $96.0 \%$ & 5.06 & 0.11 \\
\hline PLD $5 \mathrm{~mm}$ & $93.8 \%$ & $65.9 \%$ & $50.0 \%$ & $96.7 \%$ & 2.76 & 0.09 \\
\hline SLD $4 \mathrm{~mm}$ & $81.3 \%$ & $46.6 \%$ & $35.6 \%$ & $87.2 \%$ & 1.53 & 0.40 \\
\hline $\begin{array}{l}\text { Abbreviations: pN+ = positive neck-node, PPV = positive predictive value, NPV = negative predictive } \\
\text { value, LR+ = positive likelihood ratio, LR- = negative likelihood ratio, aTT = MRI tumor thickness in } \\
\text { axial view, cTT = MRI tumor thickness in coronal view, sTT = MRI tumor thickness in sagittal view, } \\
\text { PLD = paralingual distance and SLD = sublingual distance }\end{array}$ & & & & \\
\hline
\end{tabular}


Table 3

Univariate and multivariate analysis to identify potential predictor in predicting regional lymph node metastasis

\begin{tabular}{|c|c|c|c|c|c|}
\hline & & Univariate analysis & & Multivariate ar & \\
\hline & & OR $(95 \% \mathrm{Cl})$ & $\begin{array}{l}P \\
\text { Value }\end{array}$ & OR $(95 \% \mathrm{Cl})$ & $\begin{array}{l}P \\
\text { Value }\end{array}$ \\
\hline Age & $\leq 60$ & Reference & 0.209 & - & - \\
\hline & $>60$ & $0.433(0.137-1.376)$ & & & \\
\hline Gender & Male & Reference & 1.000 & - & - \\
\hline & Female & $0.957(0.390-2.351)$ & & & \\
\hline Tumor size & T1 & Reference & 0.000 & Reference & 0.024 \\
\hline & $\mathrm{T} 2$ & $\begin{array}{l}59.933(7.797- \\
460.704)\end{array}$ & & $\begin{array}{l}15.175(1.436 \\
160.329)\end{array}$ & \\
\hline Tumor location & Anterior/ & & 0.003 & & 0.011 \\
\hline & Middle & Reference & & Reference & \\
\hline & Posterior & $\begin{array}{l}5.395(2.259- \\
12.888)\end{array}$ & & $\begin{array}{l}6.311(1.514- \\
26.304)\end{array}$ & \\
\hline rTTT* & MinimalrTTT & Reference & 0.000 & Reference & 0.002 \\
\hline & SignificantrTTT & $\begin{array}{l}34.067(10.407- \\
111.518)\end{array}$ & & $\begin{array}{l}11.528(2.483 \\
53.530)\end{array}$ & \\
\hline PLD & $>5 \mathrm{~mm}$ & Reference & 0.000 & Reference & 0.007 \\
\hline & $\leq 5 \mathrm{~mm}$ & $\begin{array}{l}29.000(6.486- \\
129.668)\end{array}$ & & $\begin{array}{l}11.976(1.981 \\
72.413)\end{array}$ & \\
\hline SLD & $>4 \mathrm{~mm}$ & Reference & 0.006 & - & - \\
\hline & $\leq 4 \mathrm{~mm}$ & $\begin{array}{l}3.780(1.417- \\
10.088)\end{array}$ & & & \\
\hline Cell- & Well & Reference & 0.681 & - & - \\
\hline & Moderate/ & $1.241(0.552-2.792)$ & & & \\
\hline & Poor & & & & \\
\hline $\begin{array}{l}\text { Abbreviations: } \\
\text { SublingualDista } \\
\text { groups:"minima } \\
\text { exceed their cut } \\
\text { aTT, cTT or sTT } \\
\text { their cutoff valu } \\
\text { (either aTT, cTT }\end{array}$ & $\begin{array}{l}=\text { odds ratio, } \mathrm{Cl}= \\
\mathrm{e} \text { * } \mathrm{rTTT} \text { (radiologic } \\
\text { and " significant" } \\
\text { values. Minimalr } \\
\text { ound to be greater } \\
\text { whereas significa } \\
\text { sTT) exceeding th }\end{array}$ & $\begin{array}{l}\text { onfidence interval, PLD } \\
\text { tumor thickness threst } \\
\text { TT, depending on how } \\
\text { TT' was defined as onl } \\
\text { han its defined cutoff } \\
\text { trTTT was defined as } \\
\text { air cutoff values. }\end{array}$ & $\begin{array}{l}\text { Paralin } \\
\text { d) was } \\
\text { lany MR } \\
\text { ne orier } \\
\text { lue or } n \\
\text { least th }\end{array}$ & $\begin{array}{l}\text { alDistance, SL } \\
\text { tegorized into } \\
\text { assessed tumo } \\
\text { ation of MRI thi } \\
\text { MRI thicknesse } \\
\text { orientations of }\end{array}$ & $\begin{array}{l}\text { esses } \\
\text { (either } \\
\text { ding } \\
\text { ckness }\end{array}$ \\
\hline
\end{tabular}




\section{Figures}
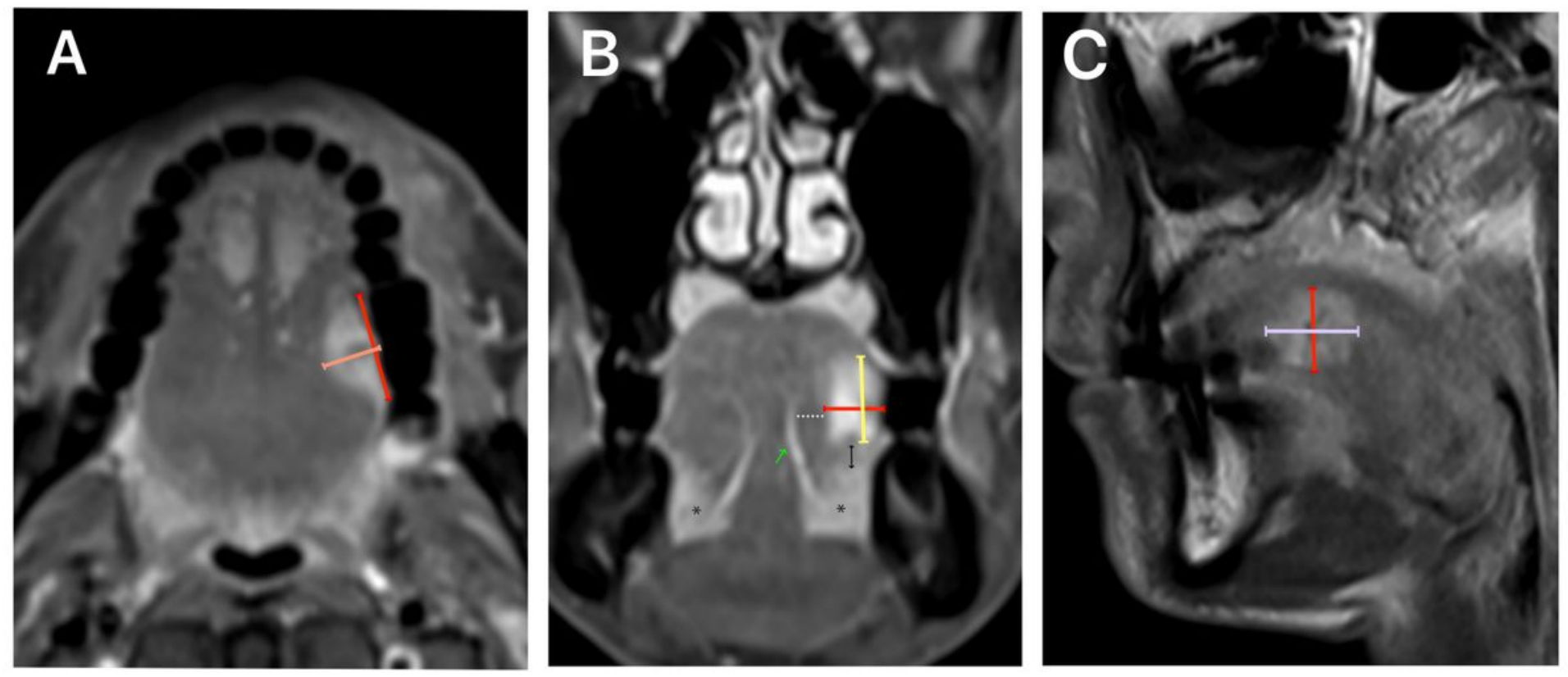

\section{Figure 1}

T1W1 MRI showed measurement of MRI-assessed tumor thickness in three dimensions All reference lines (red line) were defined as the longest tumor diameter in each direction. A. MRI-assessed tumor thickness in a mediolateral direction was defined as aTT (orange line), B. MRI-assessed tumor thickness in a vertical direction was defined as CTT (yellow line). C. MRI-assessed tumor thickness in an anteroposterior direction was also defined as STT (purple line). In the coronal view, a thin high signal intensity (green arrow line) was used to identify the deep lingual artery and inferior lateral area of high signal intensity (asterisk) was indicated the sublingual space. Paralingual distance was defined as the distance between the lateral aspect next to the deep lingual artery and medial border of the tumor (dotted line). Sublingual distance was determined by measuring the vertical distance between the inferior border of tumor and the sublingual space (black arrow line). 
A.

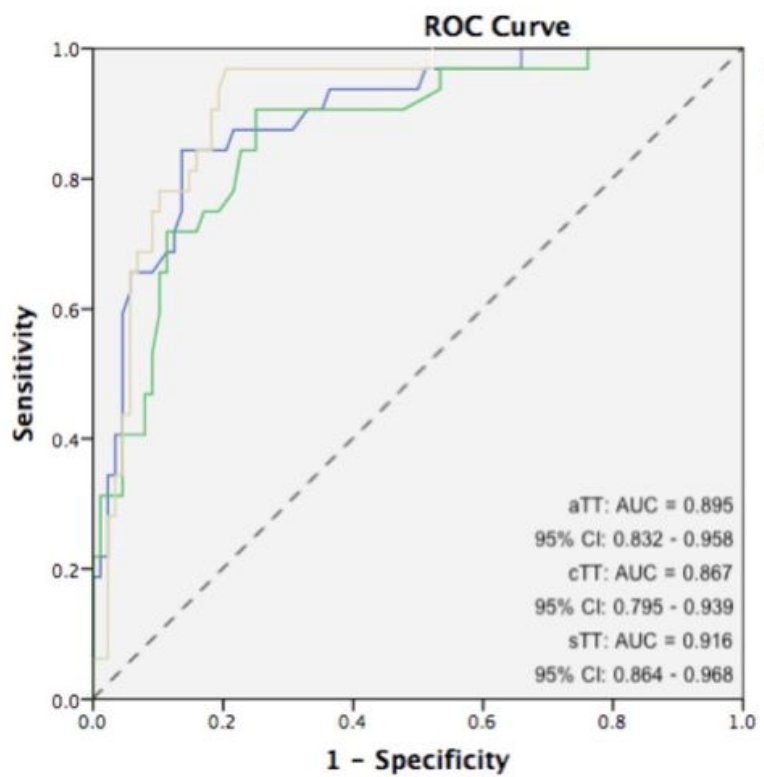

B.

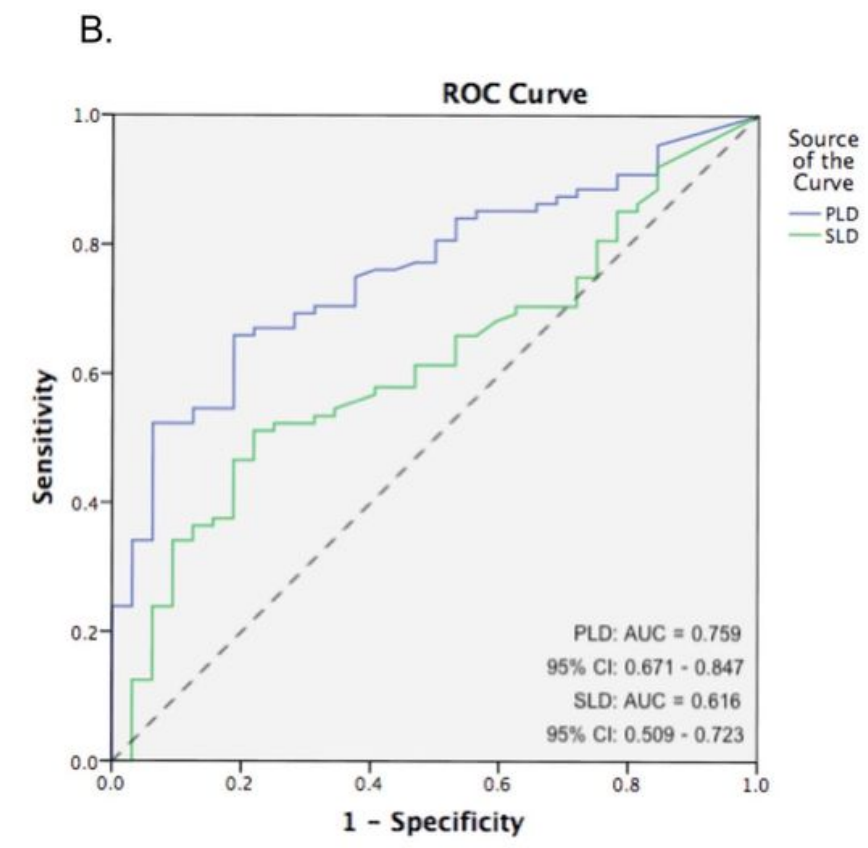

\section{Figure 2}

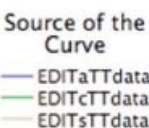

Receiver operation characteristic curve indicated the optimal cutoff value of MRI-multiparameter inevaluating cervical metastasis A. Area under the curve (AUC) of MRI-assessed tumor thickness cutoff values were a statistically significant difference $(P<0.05)$; aTT cutoff value as $8.5 \mathrm{~mm}$ was 0.895 (blue line), AUC of cTT cutoff value as $11 \mathrm{~mm}$ was 0.867 (green line) and AUC of sTT cutoff value as $12 \mathrm{~mm}$ was 0.916 (yellow line). The cutoff for sTT curve being the most significantly different. B. Paralingual distance (PLD) and sublingual distance (SLD) cutoff values were $5 \mathrm{~mm}(\mathrm{AUC}=0.759)$ and $4 \mathrm{~mm}(\mathrm{AUC}=$ 0.616), respectively. 
Points

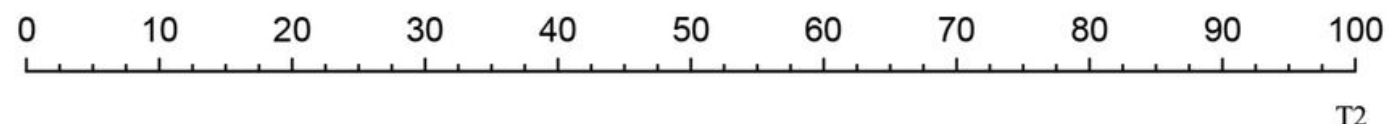

Tumor Size

Tumor Location
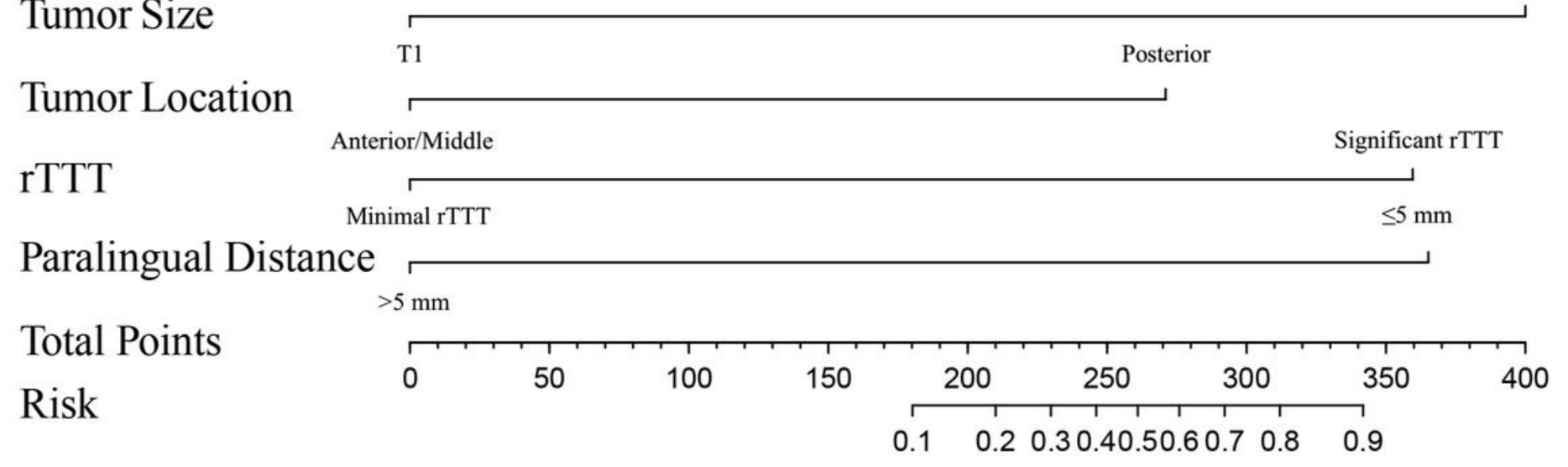

Figure 3

Preoperative nomogram incorporating multiparameter MRI for individualized occult metastasis risk prediction in early-stage SCCLT Summary of nomogram consisted of a set of scales in which each scale represents a characteristic of the study population. The each predictor to be considered as a point value based on power predictive ability in assessing occult metastasis risk. The points assigned to each of the four predictors and the total points obtained from four predictors are summed and indicated the probability of the patient likelihood of having cervical lymph node metastasis. 
A.

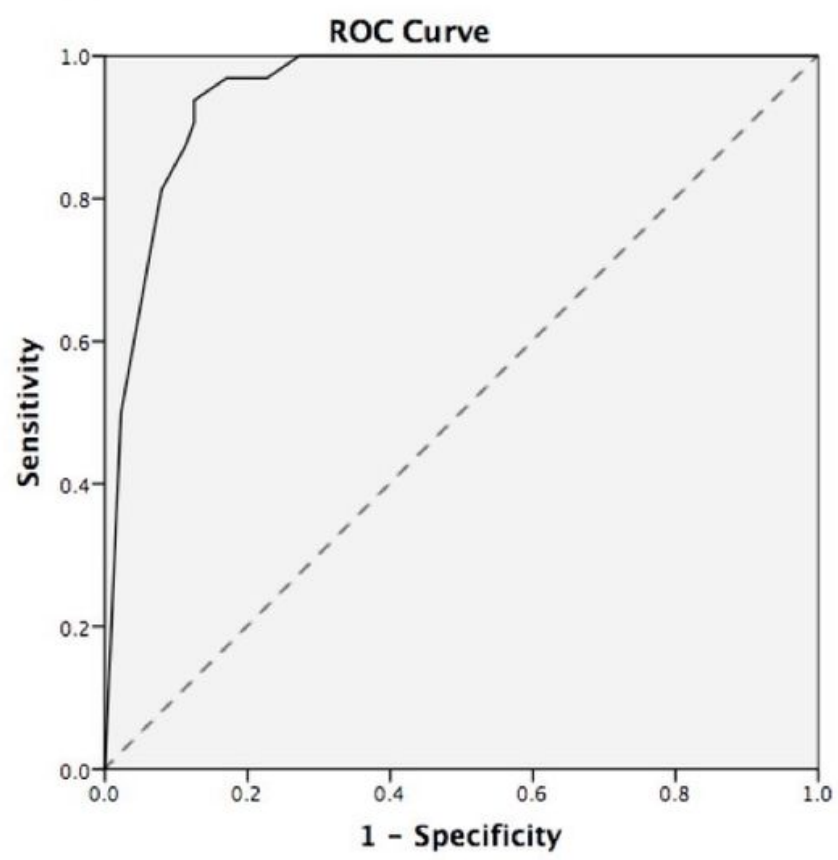

C.

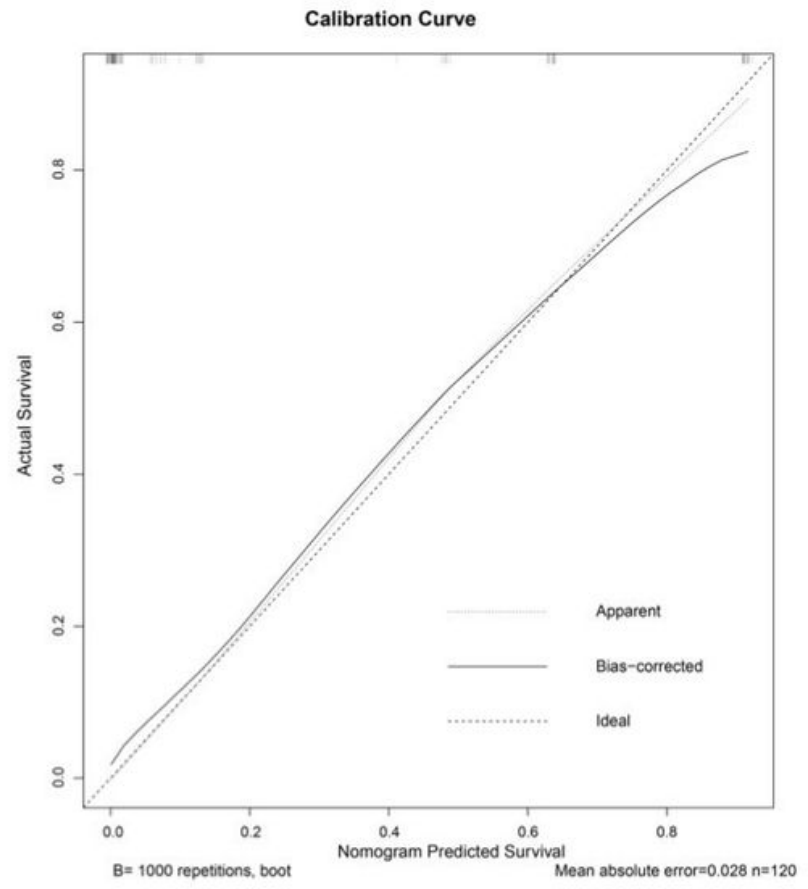

B.

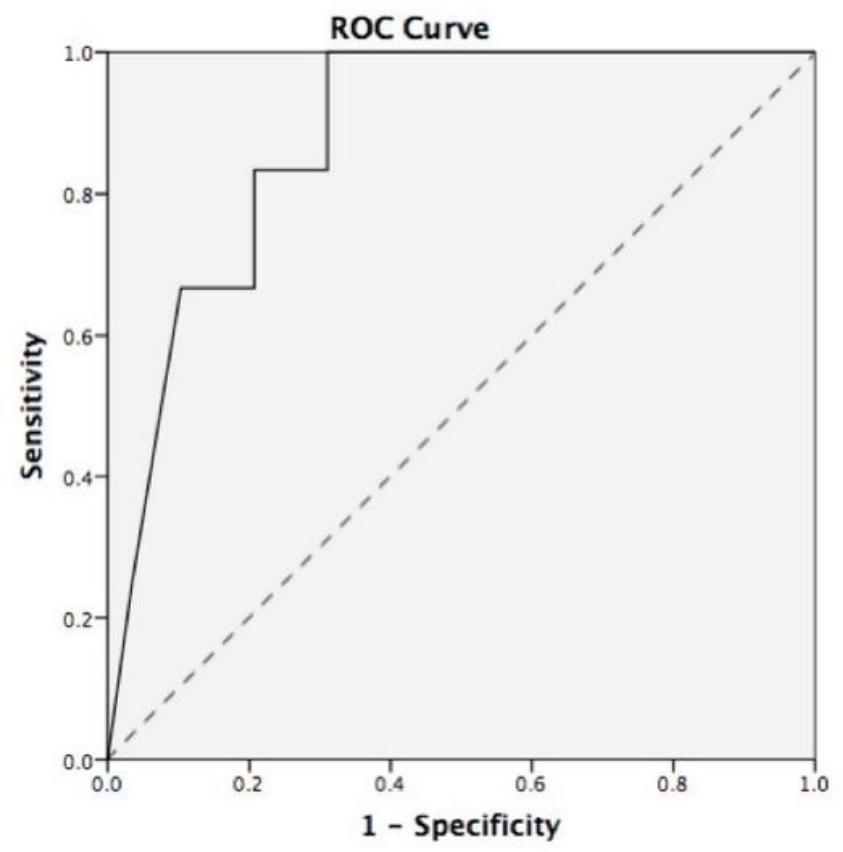

D.

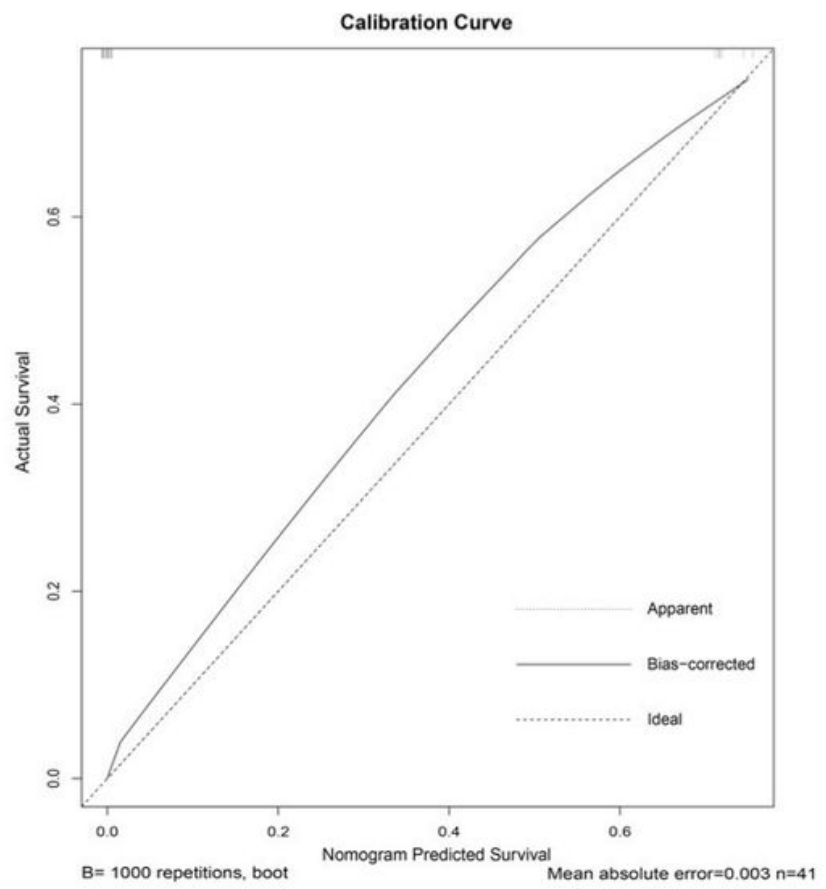

\section{Figure 4}

Prediction performance and calibration of the multiparametric MRI-based nomogram in detecting cervical lymph node metastasis The nomogram cutoff value of 210 points was used to generate the patients into metastasis risk groups in this study. Prediction performance of the multiparametric MRI-based nomogram in the primary $(A)$ and validation $(B)$ cohorts was measured by the area under the receiver operating characteristic curve. The predictive accuracy to discriminate the patients likelihood of having occult nodal metastasis were $0.952(95 \% \mathrm{Cl} 0.917-0.987, \mathrm{P}<0.001)$ in the primary cohort and 0.881 (95\% 
$\mathrm{Cl} 0.779-0.983(\mathrm{P}<0.001)$ in the validation cohort. Calibration curve of the MRI-based nomogram for predicting the risk of occult nodal metastasis in the primary (C) and validation (D) cohorts of patients with early-stage SCCLT. Calibration curve indicated there had no apparent departure from perfect fit, with good correlation between the prediction and observation in both cohorts. 